\title{
Mini-review on "A novel one-step purification of mouse factor IX"

Article Info

\section{Article Notes}

Received: August 03, 2016

Accepted: August 23, 2016

\section{${ }^{*}$ Correspondence:}

Dr. Rinku Majumder,

Department of Biochemistry \& Molecular Biology, LSU Health Science Center, 1901 Perdido Street, MEB-7114, New Orleans, LA-70112

E-mail: rmajum@|suhsc.edu

( 2016 Rinku Majumder. This article is distributed under the terms of the Creative Commons Attribution 4.0 International License.

\section{Keywords}

Factor IX (FIX)

Hemophilia B

Mouse models
Factor IX (FIX) is a 70-kDa, single-chain, vitamin K-dependent glycoprotein present in trace amounts $(\sim 95 \mathrm{nM})$ in blood plasma ${ }^{1,2}$. FIX is synthesized as a zymogen that is converted to a serine protease, FIXa, by the activated form of factor $\mathrm{XI}^{3,4}$. FIXa has a central function in the intrinsic pathway of blood coagulation, in that it acts as an activator of factor X (FX), directly upstream of the common pathway ${ }^{1,5}$. Upon activation, FXa concomitantly converts prothrombin to thrombin to initiate the formation of the fibrin lattice $^{6,7}$.

\section{Isolation of Factor IX and Factor IXa}

Purified FIX is essential for the treatment of Hemophilia $\mathrm{B}$, and many animal model investigations, regarding possible augmentations in Hemophilia B therapy, also require abundant sources of FIX (see below). A number of FIX purification approaches have been reported, but all are either multi-step and time-consuming or of low yield ${ }^{1,2}$. Thus, the report by Pilli et al. represents a welcome advancement in meeting patient and research requirements for purified FIX. The authors describe a rapid, singlestep human FIX purification process using a IXSelect affinity column (GE Healthcare Life Sciences) ${ }^{8}$. The human IXSelect affinity medium consists of a single-chain antibody fragment directed against FIX (Data file 29-1084-88 AA; https://www.gelifesciences.com/ gehcls_images/GELS/Related\%20Content/Files/1402917750761/ litdoc29108488_20140616222347.pdf). From $100 \mathrm{~mL}$ of human plasma, the authors purified $200 \mu \mathrm{g}$ of FIX, a $30 \%$ yield similar to that of other purification methods ${ }^{9}$. Significantly, the affinity medium was also capable of isolating a substantial amount of murine FIX ( $80 \mu \mathrm{g}$ from $100 \mathrm{~mL}$ of mouse plasma). Using a modified activated partial thromboplastin time clotting assay ${ }^{10}$, the authors showed that the purified proteins were fully functional. As detailed below, the IXSelect affinity medium should find many applications in the clinic and in hemostasis research.

\section{Hemophilia B}

Hemophilia B is an X-linked bleeding disorder resulting from FIX deficiencies ${ }^{11}$. More than 1000 different genomic mutations are known to cause Hemophilia B, affecting an estimated 20,000 people in the US, alone ${ }^{12-14}$. Current treatment consists of intravenous infusion of recombinant or plasma-derived FIX ${ }^{15}$, both of which are subject to rapid turnover following injection; prophylactic dosing is required, approximately every 3-7 days, making abundant sources of inexpensive FIX essential to the economic treatment of patients. 
The human IXSelect affinity medium was designed specifically for purification of FIX in industrial processes at large scale. Human IXSelect has a capacity of 6 mg FIX/ $\mathrm{mL}$ medium. The agarose-based matrix is highly crosslinked and rigid, which allows for high flow rates and rapid processing of large sample volumes. The resin can be used indefinitely, provided proper use, regeneration, and storage protocols are employed. Thus, IXSelect would appear to be an ideal medium for supplying the FIX needed for Hemophilia B individuals, and the report by Pilli et al. substantiates that notion. Furthermore, the resin is devoid of any other mammalian component (the antibody fragment ligand is produced in yeast), thereby satisfying FDA regulatory conditions.

As indicated above, Hemophilia B is treated with either plasma-derived or recombinant FIX. Plasma FIX, synthesized in the liver as the zymogen, requires a number of critical post-translational modifications, in addition to proteolytic processing, before it is secreted as a functional protease. The most notable post-translational modification is the gamma $(\gamma)$-carboxylation of $12 \mathrm{~N}$-terminal glutamic acid residues to form $\gamma$-glutamic acid $(7,9)$; this $\mathrm{N}$-terminal region, referred to as the Gla domain, is involved in membrane binding and is required for activation.

Although plasma FIX is fully functional, purification of the protein from plasma may entail a risk for the transmission of infectious agents upon administration to Hemophilia B individuals. Thus, recombinant FIX is now the most often prescribed form, as a safer and more economic alternative therapy for Hemophilia B (7). However, production of completely $\gamma$-carboxylated recombinant human FIX protein, in high yield, has been challenging ${ }^{16}(8)$. To obtain fully modified recombinant FIX, Jianming et al. ${ }^{17}$ engineered a $\mathrm{CHO}$ cell line that co-expresses FIX with the necessary modification enzymes ( -glutamyl carboxylase, vitamin $\mathrm{K}$ epoxide reductase, and furin/PACE cleaving enzyme). The product, BeneFix (Wyeth), contains an average of 11.5 $\gamma$-carboxyglutamic acid residues; full FIX activity requires $\gamma$-carboxylation of only the first 10 Glu residues. BeneFix is purified with four chromatographic steps and subjected to nanofiltration to remove potential viral contaminants ${ }^{18}$. The GE data bulletin referred to previously describes IXSelect purification of FIX from a CHO cell lysate, although it is not reported whether the cell line is the same as that engineered by Jianming et al.

\section{Animal studies}

Hemophilia B has an extensive history of study using animal models, and new therapeutics ${ }^{3,15}$ for Hemophilia B, which are being explored with such animal models, often require large amounts of purified experimental versions of FIX. Thus, the most important outcome of the study by Pilli et $\mathrm{al}$. is the finding that the IXSelect affinity medium can efficiently purify non-human FIX. This result is impressive, given a $82 \%$ sequence similarity between mouse and human factor IX. Importantly, human and murine FIX interact with the same proteins in each system, resulting in similar behaviors in a range of coagulation assays common in the clinical setting ${ }^{15}$. Hence, the mouse is a useful FIX model for human coagulation therapeutics, particularly for the ability to modify its genetics ${ }^{19}$ and for when large groups of genetically defined strains are to be studied. Murine models, currently in use, include mice deficient in FIX and mice engineered to express FIX variants ${ }^{20}$.

Canine and primate models of Hemophilia B are also in use for testing the efficiency of scaling up therapeutics for long-term follow up studies, and for studies requiring larger blood volumes ${ }^{15,21,22}$. Primate models are especially useful, because the animals are most similar to humans in size and anatomy ${ }^{23,24}$. Thus, it will be of interest to determine the efficacy of the IXSelect medium in isolation of FIX from other species. One would expect the medium to perform well with chimpanzee and macaque FIX, which share with human FIX 99.8\% and 96.4\% amino acid identity, respectively. Considering the result with mouse plasma, other sources of FIX that will likely be amenable to IXSelect purification are dog (86\% identity), cow (84\% identity), pig ( $86 \%$ identity), rat ( $82 \%$ identity), and rabbit (83\% identity). To compare efficiencies of purification, it will be necessary to determine the circulating concentrations of FIX in the plasmas of non-human species. Also, as noted above for human FIX, Pilli et al. initially reported a $30 \%$ yield from the IXSelect medium; this yield may be limited by the nature of the antibody fragment ligand used as the affinity medium, but perhaps some optimization testing to boost yield is warranted. Efficient FIX isolation from multiple sources is essential for assays in different organisms and, ultimately, for the progression to human clinical trials.

\section{Future applications}

The highly specific IXSelect column and media, tagged with a humanized FIX antibody, previously held promise for the production of large yields of pure human FIX from industrial-sized sample volumes ${ }^{25}$. The results of Pilli et $a l^{8}$ should extend the applicability of the column to the high yield purification of FIX from the plasmas of a wide range of mammals that are frequently used to test Hemophilia therapeutics. Furthermore, the authors have readily isolated multiple highly pure, mutant forms of FIX, which have been characterized by in vitro activity assays (unpublished results). These mutant proteins are vital for identifying the means by which FIX interacts with other proand anti-coagulant factors ${ }^{8}$. Similarly, the $>1000$ mutations already known to affect human FIX activity and abundance represent a rich pool of targets for identification of small molecule therapeutics; screening for such compounds will likely require isolation of numerous mutants in 
recombinant form, which will be greatly facilitated by the simple, economical, and rapid one-step IXSelect column.

Finally, BeneFix has been in use since 1997, and it is the only FDA-approved recombinant FIX. Notably, the amount of FIX secreted by engineered CHO cells is relatively low, about $30-40 \mathrm{mg} / \mathrm{L}^{26}$. Thus, transgenic animals that secrete FIX from mammary glands are being explored as sources of more abundant recombinant FIX. One of the most successful systems is pig, which has been reported to secrete $375 \mathrm{mg}$ FIX/L of milk ("reported FIX secretion values, corrected to actual concentration of active form" ${ }^{26}$ ), with a native degree of specific activity and complete carboxylation ${ }^{27}$. It will be interesting to test the efficacy of the IXSelect medium in purification of FIX from milk.

In conclusion, the IXSelect medium should continue to show its range of applicability in purification of wildtype and mutant variants of FIX from numerous cellular environments. Less expensive and more efficient isolation of these proteins is advantageous towards bringing Hemophilia B studies to the human level. The ease of FIX isolation, described by Pilli et al., should provide a significant enhancement in the use of multiple animal models in the future development of new therapeutic agents for Hemophilia B.

\section{References}

1. Andersson LO, Borg H, Miller-Andersson M. Purification and characterization of human factor IX. Thromb Res. 1975;7(3):451-459.

2. Osterud B, Flengsrud R. Purification and some characteristics of the coagulation factor IX from human plasma. Biochem J. 1975;145(3):469474.

3. Pipe SW. Hemophilia: new protein therapeutics. Hematology Am Soc Hematol Educ Program. 2010;2010:203-209.

4. Gailani D, Geng Y, Verhamme I, et al. The mechanism underlying activation of factor IX by factor XIa. Thromb Res. 2014;133 Suppl $1:$ S48-51.

5. Chung KS, Madar DA, Goldsmith JC, et al. Purification and characterization of an abnormal factor IX (Christmas factor) molecule. Factor IX Chapel Hill. J Clin Invest. 1978;62(5):1078-1085.

6. Walker RK, Krishnaswamy S. The activation of prothrombin by the prothrombinase complex. The contribution of the substrate-membrane interaction to catalysis. J Biol Chem. 1994;269(44):27441-27450.

7. Krishnaswamy S. Prothrombinase complex assembly. Contributions of protein-protein and protein-membrane interactions toward complex formation. J Biol Chem. 1990;265(7):3708-3718.

8. Pilli VS, Plautz WE, Monroe DM, et al. A novel one-step purification of mouse factor IX. Thromb Res. 2016;139:125-126.

9. Bajaj SP, Rapaport SI, Prodanos C. A simplified procedure for purification of human prothrombin, factor IX and factor X. Preparative biochemistry. 1981;11(4):397-412.

10. Chattopadhyay R, Sengupta T, Majumder R. Inhibition of intrinsic Xase by protein $\mathrm{S}$ : a novel regulatory role of protein $\mathrm{S}$ independent of activated protein C. Arterioscler Thromb Vasc Biol. 2012;32(10):23872393.

11. Liebman HA, Limentani SA, Furie BC, et al. Immunoaffinity purification of factor IX (Christmas factor) by using conformation-specific antibodies directed against the factor IX-metal complex. Proc Nat Acad Sci U S A. 1985;82(11):3879-3883.

12.CHRISTMAS disease. Br Med J. 1952;2(4799):1405-1406.

13. Mannucci PM, Tuddenbam EG. The hemophilias: progress and problems. Semin Hematol. 1999;36(4 Suppl 7):104-117.

14. Mannucci PM, Tuddenham EG. The hemophilias--from royal genes to gene therapy. N Engl J Med. 2001;344(23):1773-1779.

15. Sabatino DE, Nichols TC, Merricks E, et al. Animal models of hemophilia Prog Mol Biol Transl Sci. 2012;105:151-209.

16. Bond M, Jankowski M, Patel $\mathrm{H}$, et al. Biochemical characterization of recombinant factor IX. Seminars in hematology. 1998;35(2 Suppl 2):11-17.

17.Liu J, Jonebring A, Hagstrom J, et al. Improved expression of recombinant human factor IX by co-expression of GGCX, VKOR and furin. The protein journal. 2014;33(2):174-183.

18. Harrison S, Adamson S, Bonam D, et al. The manufacturing process for recombinant factor IX. Seminars in hematology. 1998;35(2 Suppl 2):4-10.

19. Emeis JJ, Jirouskova M, Muchitsch EM, et al. A guide to murine coagulation factor structure, function, assays, and genetic alterations. J Thromb Haemost. 2007;5(4):670-679.

20.Sarkar G, Koeberl DD, Sommer SS. Direct sequencing of the activation peptide and the catalytic domain of the factor IX gene in six species. Genomics. 1990;6(1):133-143.

21. Nichols TC, Dillow AM, Franck HW, et al. Protein replacement therapy and gene transfer in canine models of hemophilia A, hemophilia B, von willebrand disease, and factor VII deficiency. ILAR J. 2009;50(2):144-167.

22.Lozier JN, Nichols TC. Animal models of hemophilia and related bleeding disorders. Semin Hematol. 2013;50(2):175-184.

23. Chandler RJ, Venditti CP. A barrel of monkeys: scAAV8 gene therapy for hemophilia in nonhuman primates. Mol Ther. 2011;19(5):826-827.

24.Feng D, Stafford KA, Broze GJ, et al. Evidence of clinically significant extravascular stores of factor IX. J Thromb Haemost. 2013;11(12):21762178.

25. Hagel L, \& GJ, Sofer G. Development, Manufacturing, Validation and Economics. Handbook of Process Chromatography. 2008;Second Edition.

26. Orlova NA, Kovnir SV, Vorobiev, II, et al. Coagulation Factor IX for Hemophilia B Therapy. Acta naturae. 2012;4(2):62-73.

27.Van Cott KE, Butler SP, Russell CG, et al. Transgenic pigs as bioreactors: a comparison of gamma-carboxylation of glutamic acid in recombinant human protein $\mathrm{C}$ and factor IX by the mammary gland. Genetic analysis : biomolecular engineering. 1999;15(3-5):155-160. 